\title{
Theory and extragalactic masers
}

\author{
William D. Watson \\ Department of Physics, University of Illinois, Urbana, IL 61801-3080
}

\begin{abstract}
Theoretical research on extragalactic masers (and hence this review) is almost entirely directed toward $22 \mathrm{GHz}$ water masers in circumnuclear disks with the focus being the near ideal masing disk at the nucleus of the galaxy NGC4258. The discussion here is organized around (1) the excitation and conditions for the masers, (2) the spatial and spectral appearance of the maser emission, and (3) the mass accretion rate and structure of the disk. In addition, a summary is given of the basic physics (spectral linebreadths and maser polarization) that underlies certain interpretations of these and other astrophysical masers.
\end{abstract}

\section{Introduction}

Although there are some thirty galaxies in which $\mathrm{H}_{2} \mathrm{O}$ masers are observed (certain others have $\mathrm{OH}$ masers), there is little in the way of "theory" other than for the few where there is evidence for disk structure. Even for these, almost all of the theoretical activity has been directed toward NGC4258 where the highly refined observations of $22 \mathrm{GHz}$ water masers in a near ideal, thin Keplerian disk provide the best opportunity for applying and testing ideas. My discussion of the "Theory and Extraglactic Masers" is thus largely a summary of theory directed toward the masers in a single galaxy-but it is a paradigm of considerable importance, and the ideas involve the basic description of molecular accretion disks near active galactic nuclei. Analysis of the structure of this disk utilizing information from maser observations potentially provides opportunities to assess the mass accretion rate which, when related to the energy emitted from this nucleus in other wavebands (X-ray, infrared), bears on current issues concerning active galactic nuclei-especially, on the possibility for advection dominated accretion flows. Such analysis also may help clarify the nature of the long sought mechanism for viscosity in accretion disks. Further, the masing disk in NGC4258 provides arguably the best evidence for massive black hole at the nucleus of an active (or at least mildly active) galaxy. See Moran, Greenhill, \& Herrnstein (1999) for a summary of the observational data about extragalactic $\mathrm{H}_{2} \mathrm{O}$ masers.

\section{The environment and excitation of circumnuclear water masers}

Masing by the water molecule at $22 \mathrm{GHz}$ involves states $\left(6_{16}\right.$ and $\left.5_{23}\right)$ which lie some $600 \mathrm{~K}$ above the ground state. Elevated temperatures and relatively high gas densities are thus required to create strong $22 \mathrm{GHz}$ masers. Gas kinetic temperatures of $400 \mathrm{~K}$ and hydrogen densities of $10^{9} \mathrm{~cm}^{-3}$ are considered representative for galactic water masers, though somewhat lower temperatures 
(300K) and densities $\left(10^{8} \mathrm{~cm}^{-3}\right)$ may be adequate in circumnuclear disks where the dimensions of the masing regions can be larger. Since the earliest calculations (Schmeld, Strelnitskii, \& Muzylev 1976), galactic water masers other than the circumstellar variety are generally believed to be associated with shock waves. Shock waves almost certainly create some of the environments for the extragalactic masing of water, as well. However, the accretion disks are likely to be relatively quiescent. Further, strong X-ray emission is typical at the nuclei of these galaxies. Hence, the elevated temperatures in the gas of the accretion disk that are necessary for masing have seemed most likely to be a result of heating by X-rays, though shock waves (Maoz \& McKee 1998) and even viscous heating (Desch, Wallin, \& Watson 1998) cannot readily be dismissed.

The chemistry and excitation for masing in a dense gas on which X-rays with intensities that are likely in circumnuclear environments has been computed by Neufeld, Maloney \& Conger (1994). As in galactic water masers, thermal collisions elevate the water molecules to a range of excited states. The inversion results from the ensuing radiative/collisional cascade. Escape of a fraction of the infrared, spectral line radiation that is involved in the cascade is essential to prevent the thermalization of the populations of the masing states, and hence the quenching of the masing. In standard calculations including those of Neufeld et al. (1994), the escape (and hence the maser power) is limited by the trapping due to reabsorption of the infrared radiation by the water molecules themselves (see Figure 1). However, the maser power can be increased significantly by utilizing Deguchi's (1981) recognition that absorption of the infrared radiation by dust grains can serve as an alternative to escape for preventing the thermalization of the masing transition. The results of calculations (Collison \& Watson 1995) for the pumping that include the influence of this absorption by dust grains in the circumnuclear masing environments created by X-rays are shown in Figure 1. It is essential that the temperature of the grains remain below that of the gas. This requirement is satisfied for a wide range of likely conditions (Desch et al. 1998). Although the potentially important issue of time variations in the flux from extragalactic masers is beyond the scope of this discussion, it is noteworthy that changes in the temperatures of the dust grains may play a role in understanding the short time scales for the changes (Neufeld 2000; Gallimore et al. 2001).

\section{Masing in a keplerian disk and its relationship to the observations}

From the viewpoint of a distant observer in the plane of a masing Keplerian disk that is uniformly pumped in a ring between inner radius $R_{i}$ and outer radius $\mathrm{R}_{o}$, the optical depths for unsaturated masing are largest toward the center of the disk and for rays at the sides of the disk with impact parameters that are near (but slightly less than) the outer radius of the disk. The gradients in velocity are a minimum in these directions. The emission at the sides arises mainly from locations along the rays where the rays are nearly tangent to the Keplerian orbits (i.e., near the midlines). Thus, three symmetrical peaks in the spectrum and three images extended along a line in the sky are considered indicative of a disk. If the masing is highly saturated, the optical depths at the sides will be largest for rays with impact parameters near the inner radius of the masing ring and will occur at Doppler velocities that are essentially equal 


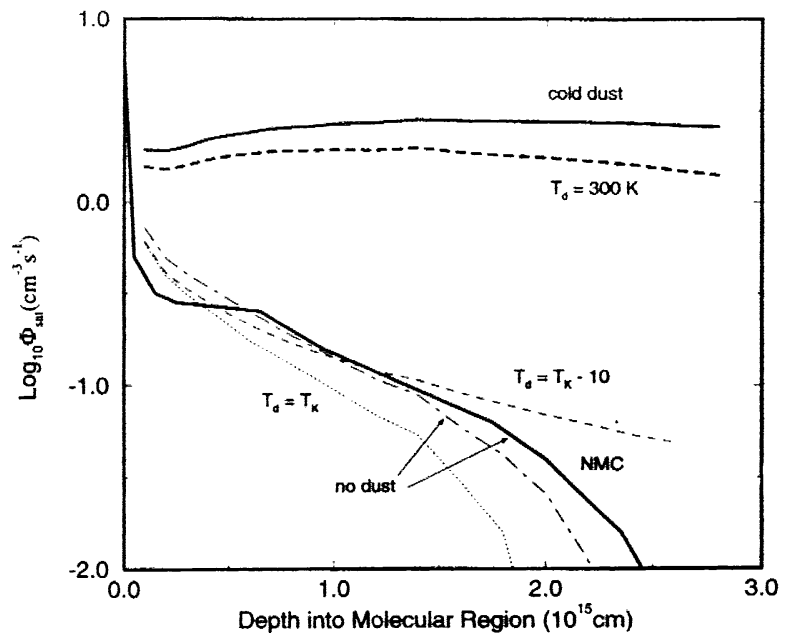

Figure 1. The log of the maser photon emissivity vs distance into a slab of gas that is irradiated by an X-ray flux similar to that expected for the circumnuclear masing gas in the galaxy NGC4258. The expected enhancement due to dust grains is best seen by comparing the lines labelled "cold dust" and " $\mathrm{T}_{d}=300 \mathrm{~K}$ " with the solid line labeled NMC (Neufeld, Maloney, \& Conger 1994) from the calculation in which the effects of dust are not included. From Collison \& Watson (1995; also Watson \& Wallin 1997).

to the velocities of the Keplerian orbits that are tangential to these rays. Toward the center, the Doppler velocities of the most intense rays at each impact parameter will vary linearly with the impact parameter. This variation is proportional to the rotational velocity at the outer edge of the ring if the masing is unsaturated, and is proportional to the rotational velocity at the inner edge if the masing is highly saturated. In addition to exhibiting characteristics similar to the foregoing, the Doppler velocities of a number of microfeatures within the central emission of NGC4258 are observed to change linearly with time (Baan, Haschick, \& Peng 1993). As a result, the diverse data from NGC4258 can be interpreted as defining a masing disk viewed nearly edge-on and the mass of the object at its center can be inferred (Watson \& Wallin 1994). In more recent observations, images of these masing features have been observed to move across the sky. For NGC4258, the maser amplifications toward the center and at the sides are not at all similar. Emission toward the center is prominent, but only because the masing here is amplifying a strong continuum source at the center. Clearly, the clumpy appearance of the maser emission from NGC4258 indicates some form of irregularities in the medium of the disk. At the same time, the medium must be smooth enough (or the irregularities numerous enough) that emission at the sides occurs mainly near the midlines where the lengths for coherence in velocity are greatest. Widely separated, but "aligned" masers can be quite intense (Deguchi \& Watson 1989).

Turbulence is prevalent in astrophysical environments of diffuse matter (e.g., supersonic turbulence in molecular clouds). MHD turbulence is believed to be 


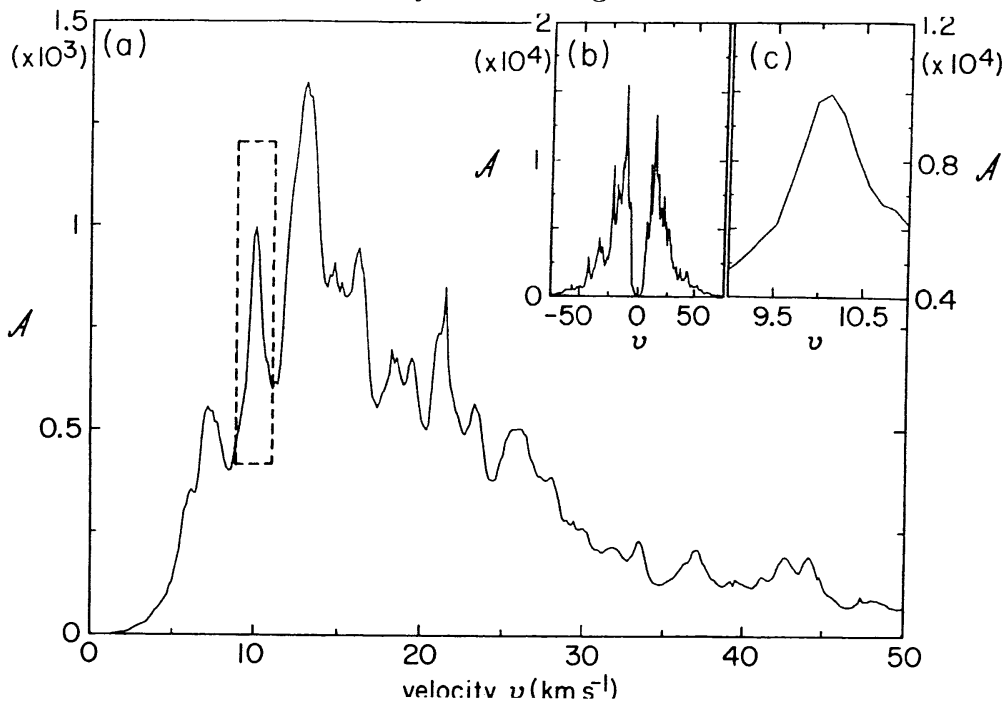

Figure 2. A representative spectrum from the simulations of a turbulent, masing Keplerian disk that is intended to describe the central emission from the circumnuclear disk in NGC4258. (a) An expanded view to show the microstructure of the full spectrum in panel (b). The feature within the dashed rectangle is expanded further in panel (c) to exhibit the narrow breadth of the feature. The spectrum (versus Doppler velocity) is presented in terms of the factor by which the background continuum source is amplified by the masing. From Wallin, Watson, \& Wyld (1998).

involved in the viscous dissipation mechanism for accretion disks. It is thus natural to inquire as to whether the rather gentle irregularities in the optical depths that are caused by turbulence of a "generic" form are sufficient to create spectra that are similar to those that are observed for NGC4258. We have thus performed computations that utilize standard methods to create representative turbulent velocity fields in the gas of a disk with dimensions and with Keplerian velocities similar to those of the NGC4258 disk. For simplicity, the maser opacity is taken as constant and maser saturation is ignored. Examples of spectra from our computations are given in Figures 2 and 3 for rms turbulent velocities that are equal to the sound speed. What is shown in these Figures is the maser amplification due to the disk. We have reasoned that the magnitudes of the amplification factors in these Figures, when combined with best estimates for the intensities of the background continuum radiation that is being amplified, are approximately what is required to yield the observed fluxes. These spectra can be compared with observations in, e.g., Myoshi et al. (1995).Rms turbulent velocities that are approximetely equal to the sound speed in the masing gas seem to be necessary to obtain spectra that are similar to the observed spectra. Deguchi (1982) originally demonstrated that representative turbulent velocity fields obtained in a somewhat analogous way lead to images for circumstellar masers that are similar to the observed images (also, Sobolev, Wallin, \& Watson 1998 for spectra and images of methanol masers in a turbulent medium).

The possibility for a quite different origin of the masing features at the sides of the disk has been recognized by Maoz \& McKee (1998). The reasoning in this interpretation is that the mass of the disk may be within the range where the Toomre stability parameter $Q$ indicates that spiral density waves will form. 


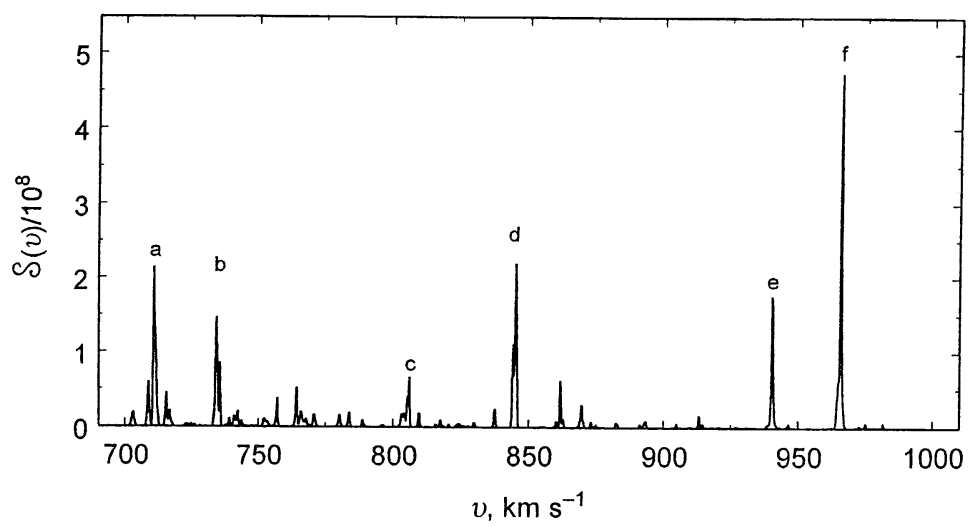

Figure 3. Similar to Figure 2 except that the spectrum here is intended to describe the maser emssion from the side of the disk. The meaning of the amplification factor is analogous to that in Figure 2 though its magnitude is quite different because these masers are not amplifying a strong continuum source. From Wallin, Watson \& Wyld (1999).

The features at the sides are then proposed to occur where the lines of sight are tangent to these spirals. Shocks form at the edges of these waves where gas flows into the wave, and provide the conditions for the water masers. Because the pattern of the waves is rotating, the locations where the tangents occur drift outward in radius to lower Keplerian rotational velocities. An acceleration of the Doppler velocities of the masing features is thus predicted. The absence of such accelerations in refined observations (Bragg et al. 1999) is in conflict with the proposed interpretation - at least for what seem to be the most likely choices for the relevant parameters. In a somewhat related idea but directed toward the presumed masing disk of NGC1068, Kumar (1999) describes how gravitational instabilities in the disk can create the masing clumps when the mass of the disk is larger than that for which spiral density waves are expected. Other discussions of the nature of the masing disk include Kartje, Konigl, \& Elitzur (1999), and Babkovskaya \& Varshalovich (2000). Novel aspects of radiative processes also have been examined (Deguchi 1994; Gangadahara, Deguchi, \& Lesch 1999).

A pervasive issue in understanding and interpreting the observations of astrophysical masers is the degree of saturation. To proceed directly from the observed flux, one must resort to highly uncertain estimates for the beaming angle-ordinarily utilizing a cylindrical geometry for the masing region. For Keplerian disks, the cylindrical approximation tends to overestimate the degree of saturation (Watson \& Wyld 2000). Nevertheless, we and others have reasoned in this way that the masers in NGC4258 are likely to be no more than partially saturated (see Wallin et al.1998). An alternative approach is to recognize that (in the absence of velocity gradients) the spectral linebreadths of unsaturated masers are narrower than the thermal breadths, and that the spectral lines re- 
broaden to the full thermal breadths when the maser becomes saturated. Despite assertions to the contrary, our own rigorous calculations (Emmering \& Watson 1994) demonstrate that the rebroadening does occur to the full Maxwellian breadth for spherical as well as for linear masers-and hence, almost surely this result is independent of geometry. At the gas kinetic temperature of $400 \mathrm{~K}$ that is ordinarily adopted as the approximate minimum for the pumping of $22 \mathrm{GHz}$ masers, the thermal linebreadth (FWHM) is $1 \mathrm{~km} \mathrm{~s}^{-1}$ for water molecules. I believe that it is far more likely that the $22 \mathrm{GHz}$ line is actually a merger of the three strongest hyperfine components of the $6{ }_{16}-5_{23}$ transition. They merge so well that the line profile can be undistorted. With the three merged hyperfine components, the minimum breadth for the fully rebroadened $22 \mathrm{GHz}$ maser line is $1.5 \mathrm{~km} \mathrm{~s}^{-1}$ (Nedoluha \& Watson 1991). Not only are the linebreadths of some of the galactic water masers with the highest fluxes less than $1.5 \mathrm{~km} \mathrm{~s}^{-1}$, they are even less than $1 \mathrm{~km} \mathrm{~s}^{-1}$ ! The prominent $1306 \mathrm{~km} \mathrm{~s}^{-1}$ feature from the side of the disk of NGC4258 has a linewidth of $1.1 \mathrm{~km} \mathrm{~s}^{-1}$. Many other microfeatures have breadths less than $1.4 \mathrm{~km} \mathrm{~s}^{-1}$ (Bragg, et al. 2000), and some are even less than $1 \mathrm{~km} \mathrm{~s}^{-1}$. Note that velocity gradients, unresolved components, etc. can increase the linebreadth, but no valid way is known to reduce the breadth other than unsaturated amplification.

The relaxation of molecular velocities, mainly through the emission and absorption of trapped, infrared spectral line radiation (Goldreich \& Kwan 1974) is a way to understand how masers can become saturated while retaining the narrow linebreadth of unsaturated masers. Then, the rebroadening of the spectral line is postponed until the rate for stimulated emission exceeds the rate for velocity relaxation. When velocity relaxation is important for $\mathrm{H}_{2} \mathrm{O}$ masers, the decay rate $\Gamma$ is reduced due to the trapping. However, the rate for velocity relaxation is approximately the value that ordinarily is adopted for $\Gamma$-the Einstein A-value for de-excitation of the molecular states by infrared emission (approximately $2 \mathrm{~s}^{-1}$ for the $22 \mathrm{GHz}$ states). Thus, the numerical value of the rate for stimulated emission at which rebroadening occurs is essentially unchanged as a result of velocity relaxation. Though this has been established in detail only for the $22 \mathrm{GHz}$ masing transition (Anderson \& Watson 1993), I expect that it is a rather general result.

\section{The accretion rate and structure of the masing disk}

A fraction (ordinarily taken as about five percent) of the rest energy of the matter that falls onto a black hole emerges as the bolometric luminosity in a standard interpretation. Typically, some ten percent of the bolometric luminosity appears as X-rays. By assuming steady-state, the accretion rate through the disk in NGC4258 can be estimated as $M \approx 1.4 \times 10^{-4} M_{\odot} y r^{-1}$. An outstanding issue for the "underluminous" nuclei in mildly active galaxies such as NGC4258 is whether this estimate is valid and the accretion rate is lower than for the more luminous, active galactic nuclei. Alternatively, the accretion rates may be similar to those in the luminous nuclei, but much of the energy fails to appear in the bolometric luminosity because it is advected into the black hole (advection dominated accretion flows, or ADAF's). The refined description of the masing disk in NGC4258 offers a possibility that valuable information can be obtained on this issue. Data at various wavelengths has been fit to an assumed 


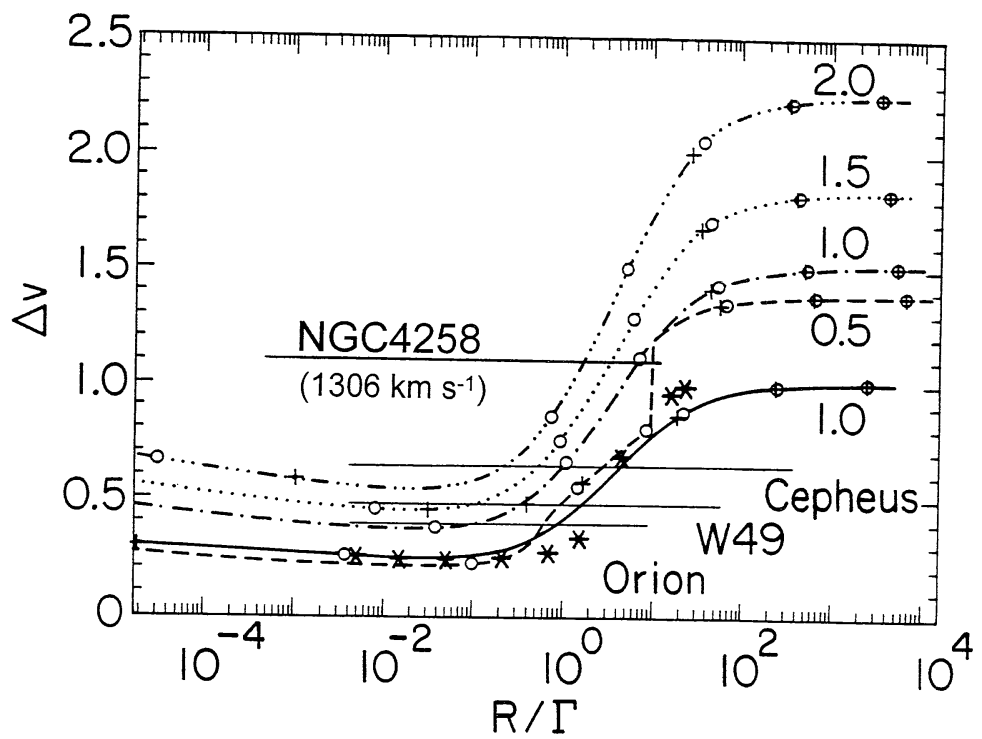

Figure 4. The linebreadth (FWHM) versus the degree of saturation for maser spectral lines. The solid line is computed for a transition between two molecular states, whereas the other lines are computed for the $6_{16}-5_{23}(22 \mathrm{GHz})$ transition of water that is assumed to be the result of the merger of the three strongest hyperfine components. The lines are labeled according to the thermal breadth of the Maxwellian distribution of the molecular velocities (note that $1 \mathrm{~km} \mathrm{~s}^{-1}$ corresponds to $400 \mathrm{~K}$ ). The computations represented by lines are performed in the idealization that the masers are linear (from Nedoluha \& Watson 1991). Also shown and indicated by asterisks are the results of similar computations for a two-level, spherical maser which demonstrate that the spectral line rebroadens in the same manner for this geometry (from Emmering \& Watson 1994). The horizontal lines indicate the linebreadths for some of the most intense flares of galactic, $22 \mathrm{GHz}$ water masers and for the prominent $1306 \mathrm{~km} \mathrm{~s}^{-1}$ feature from the side of the masing disk in NGC4258.

ADAF spectrum for NGC4258 and leads to $\dot{M} \approx 10^{-2}-10^{-3} M_{\odot} y r^{-1}$ (Lasota et al.1996; Gammie, Narayan, \& Blandford 1999; Chary et al. 2000). From the observations of the masing disk, Maoz \& McKee (1998) obtain $7 \times 10^{-3} M_{\odot} y r^{-1}$ in the spiral density wave interpretation whereas Neufeld \& Maloney (1995) find $7 \times 10^{-5} \alpha M_{\odot} y r^{-1}$ based mainly on the premise that the masing extends outward in radius until the molecules disappear and the gas becomes atomic. Here, $\alpha$ is the coefficient from the Shakura-Sunyaev description of viscosity. An approach that seems somewhat more direct involves determining the strength of the magnetic field in the disk (or a useful upper limit) by observing for the polarization of the maser radiation. Assuming a balance between the thermal and magnetic pressure within the disk, together with the Shakura-Sunyaev description of turbulence, then leads to a value for $(\dot{M} / \alpha)$. Herrnstein et al. (1998) thus infer an upper limit $10^{-2} \alpha M_{\odot} y r^{-1}$ from an upper limit of $300 \mathrm{mG}$ for the magnetic field based on their failure to detect circular polarization at a level of 1.5 percent in 
the $1306 \mathrm{~km} \mathrm{~s}^{-1}$ feature at the side of the disk. Though this limit is not sensitive enough to discriminate between an ADAF and a standard model, the prospects are good for observations of the central spectrum that are considerably more sensitive (see also Moran 2000). However, the spectrum for the circular polarization of the central emission will be quite different from that of a two-level transition if the $22 \mathrm{GHz}$ line is a merger of three hyperfine components (Watson \& Wiebe 2001) as I believe is most likely. Relating the magnetic field strength to the circular polarization probably is less difficult for isolated spectral lines (see also Nedoluha \& Watson 1992).

The mass in the disk can be related to $M_{\odot}$ utilizing the Shakura-Sunyaev prescription for the viscosity. In terms of the radius $R_{i}$ at the location of the central masers, the surface mass density at radius $R$ is (e.g., Desch et al. 1998)

$$
\Sigma=(20 / \alpha)\left(\dot{M} / 2 \times 10^{-4} M_{\odot} y r^{-1}\right)(400 K / T)\left(R_{i} / R\right)^{3 / 2} \mathrm{~g} \mathrm{~cm}^{-2}
$$

Since the X-rays from the central source can penetrate to only about $1 \mathrm{~g} \mathrm{~cm}^{-2}$, they probably fail by a considerable factor in being able to maintain the $400 \mathrm{~K}$ or so temperatures that would be required for masing by water throughout the disk - even for the $\dot{M}$ based on the "standard" interpretation of flow through the accretion disk. Thus, a cold component of the disk probably exists that is more massive than the masing component that we detect. The spectrum of NGC4258 often exhibits a narrow dip near the systemic velocity. This may be due to absorption by the cold component. Beyond the depths to which the X-rays can penetrate and excluding isolated regions where there might be localized heating, the temperature probably is less than about $50 \mathrm{~K}$. With favorable assumptions about poorly understood processes, viscous heating within the disk might be sufficient to heat a thin layer that could be the site of the observed masing (Desch et al. 1998). It could not, however, provide sufficient energy to heat the entire thickness of the disk to such temperatures. Note that heating by shocks in spiral density waves is included in this assessment of the viscous heating since the origin of the energy for both is the change in gravitational potential energy as the matter drifts inward. An estimate for the mass in the disk between the inner and outer radii at which masers are observed can readily be obtained by ignoring the variation in temperature in the above expression for $\Sigma$ and integrating to obtain

$$
M_{d i s k} \simeq\left(10^{4} / \alpha\right)\left(\dot{M} / 2 \times 10^{4} M_{\odot} y r^{-1}\right)(400 K / T) M_{\odot}
$$

The central mass about which the masing disk is orbiting has a mass of $3.6 \times 10^{7} M_{\odot}$. I am not aware of a quantitative assessment for the maximum disk mass that is consistent with the absence of observed deviations from the Keplerian rotation, but it seems unlikely to be larger than about 0.3 percent of the central mass. Based on the above $\mathrm{M}_{\text {disk }}$, there is then little if any flexibility for $\dot{M}$ to exceed the minimum value inferred from the standard description of accretion. Gravitational clumping and/or multiple thermodynamic phases may alter the simple analysis given above in essential ways. Certainly, the ShakuraSunyaev prescription for viscosity is an extreme simplification. For example, MHD simulations tend to indicate that the magnetic pressure (and hence the likely dissipation of viscous heat) decreases much less rapidly with distance away from the midplane of an accretion disk than does the gas kinetic pressure (Miller \& Stone 2000), a viewpoint that has received support from other perspectives 
(e.g., Stella \& Rosner 1984). Finally, by utilizing the Shakura-Sunyaev viscosity once more, the Toomre stability parameter $Q$ can be expressed as

$$
Q \simeq 7 \alpha(T / 400 K)^{3 / 2}\left(2 \times 10^{-4} M_{\odot} y r^{-1} / \dot{M}\right)
$$

A disk is stable for $Q \geq 1$. This marginal stability (at best) of the disk in NGC4258 underlies proposals for density waves and for the clumping of the mass. We note that an entirely different, alternative approach exists for understanding accretion in disks (Blandford \& Payne 1982), but does not lend itself to quantitative interpretations.

Acknowledgments. The author's research is supported in part by NSF Grant AST99-88104.

\section{APPENDIX - Polarization of maser radiation}

The contributions to issues of broad astrophysical importance from the observation of extragalactic masers, just as for galactic masers, often depend upon an understanding of the basic physics for the excitation and transport of astrophysical maser radiation. This is especially true in efforts to infer information about the magnetic field from the observation of the polarization of the maser radiation. Confusion about the theory for maser polarization seems to be prevalent in the literature. A summary at this time may thus be helpful. I will focus here only on the limit in which the Zeeman splitting $g \Omega$ is much smaller than the spectral linebreadth $\Delta \omega$ ("weak splitting"). This is the limit that is relevant for the circular polarization of extragalactic water masers, as well as for galactic $\mathrm{H}_{2} \mathrm{O}, \mathrm{SiO}, \mathrm{OH} 1720 \mathrm{MHz}$, and $\mathrm{CH}_{3} \mathrm{OH}$ masers. For simplicity, I will ignore any explicit consequences of cross-relaxation in this discussion. Cross-relaxation is analogous to velocity relaxation in that its main effect for polarization in the weak splitting regime is that the rate for cross-relaxation $\gamma$ replaces the "phenomenological decay rate" $\Gamma$ of the molecular populations in the discussion of this Appendix (Goldreich, Keeley, \& Kwan 1973b). However, as in the case of velocity relaxation, $\Gamma$ ordinarily is reduced when cross-relaxation is important and $\gamma$ has the approximate value that normally is associated with $\Gamma$-approximately $2 \mathrm{~s}^{-1}$ for the $22 \mathrm{GHz}$ water masers.

\section{A. The Idealization of Goldreich, Keeley and Kwan: Linear Polariza- tion}

A discussion of the theory of maser polarization naturally begins with the classic paper of Goldreich, Keeley, \& Kwan (1973a; hereafter GKK). It is therefore important to know exactly what idealized masing conditions were considered by GKK, and what answers they did and did not provide. GKK considered the basic issue of the polarization of maser radiation that emerges from a linear maser when weak, continuum seed radiation is incident at the far end of a masing region in which there is a constant magnetic field. They considered only an angular momentum $J=1-0$ masing transition and only equal pumping of the magnetic substates of $\mathrm{J}=1$. I will refer to this scenario as the "GKK idealization". The key restriction in GKK is that the solutions for the polarization given there are obtained only in limiting regimes for the maser intensity, as specified by 
the rate $R$ for stimulated emission. With $\Gamma$ as the usual decay rate for the masing states, GKK find that there is linear polarization when (a) $\Gamma \ll R \ll g \Omega$ or when (b) $g \Omega \ll R \ll(g \Omega)^{2} / \Gamma$, and that the fractional linear polarization is zero when $R \ll \Gamma$ or $(g \Omega)^{2} / \Gamma \ll R$. In regime (a), the fractional linear polarization $(\mathrm{Q} / \mathrm{I})$ is given by the widely quoted expressions $(\mathrm{Q} / \mathrm{I})=-1$ for angles $\theta$ between the magnetic field and the line-of-sight that are less than 35 degrees and for other angles between 35 and 90 degrees, $\left.(\mathrm{Q} / \mathrm{I})=3 \sin ^{2} \theta-2\right) / 3 \sin ^{2} \theta$. In regime (b), Q/I is positive and can be as large as one-third. Positive $\mathrm{Q}$ corresponds to a net linear polarization that is perpendicular to the magnetic field.

Although it accomplished a lot, the GKK analysis was incomplete even for the GKK idealization because it did not tell us quantitatively how strong the inequalities that define the various regimes must be in order for the solutions given there for the linear polarization to be applicable. More generally, the behavior of the polarization as a continuous function of maser intensity (or degree of saturation) was not provided. Several collaborative efforts at Illinois have largely completed the picture for the GKK idealization. I will now summarize the key results.

Computations for the linear polarization as a continuous function of maser intensity were initially limited to intensities for which $R \ll g \Omega$ (Western \& Watson 1984). As expected, the computations agreed with the GKK solutions in the limit of high saturation $(\Gamma \ll R)$. However, quite high saturations $R / \Gamma \approx 100$ are necessary to attain the fractional linear polarizations of 70 percent for $\mathrm{J}=1$ 0 transitions which are sometimes observed for the $\mathrm{SiO}$ masers. To attain the comparably high fractional polarization that is observed for masing of $\mathrm{SiO}$ in the $\mathrm{J}=2-1$ transition, $R / \Gamma$ must be implausibly large. It follows that linear polarization cannot be entirely due to the GKK mechanism. The next step was rephrasing the GKK density matrix equations in a way that is convenient for treating more generally the regime where $R \ll g \Omega$ is not satisfied. An additional result in this investigation was the recognition that significant linear polarization is created in regime (b) [where $g \Omega \ll R \ll(g \Omega)^{2} / \Gamma$ ] only for a J=1-0 transition (Deguchi \& Watson 1990). By integrating the radiative transfer equations that are based on the density matrix formulation, Nedoluha \& Watson (1990a) finally obtained the full solution - the fractional linear polarization for all $R$-for the GKK idealization. Complete agreement with what is expected from GKK was obtained. Answers to the question of how strong must the inequalities be in order for the GKK solutions to be applicable, and how does the linear polarization behave when they are not valid, were obtained. Representative results are reproduced in Figure 5. Note that the direction of the linear polarization is neither parallel nor perpendicular to the projection of the magnetic field on the plane of the sky when $R \simeq g \Omega$ (i.e., Stokes $\mathrm{U} \neq 0$ ).

\section{B. Additional Considerations for the Linear Polarization}

As noted above, the GKK mechanism alone of saturated masing in the presence of a magnetic field is unlikely to produce the highest fractional linear polarizations that are observed in the $\mathrm{J}=2-1$ and higher transitions of the circumstellar $\mathrm{SiO}$ masers.

Actual masing environments in astronomy may be more complicated in essential ways than the idealized environment considered by GKK. Of these, 

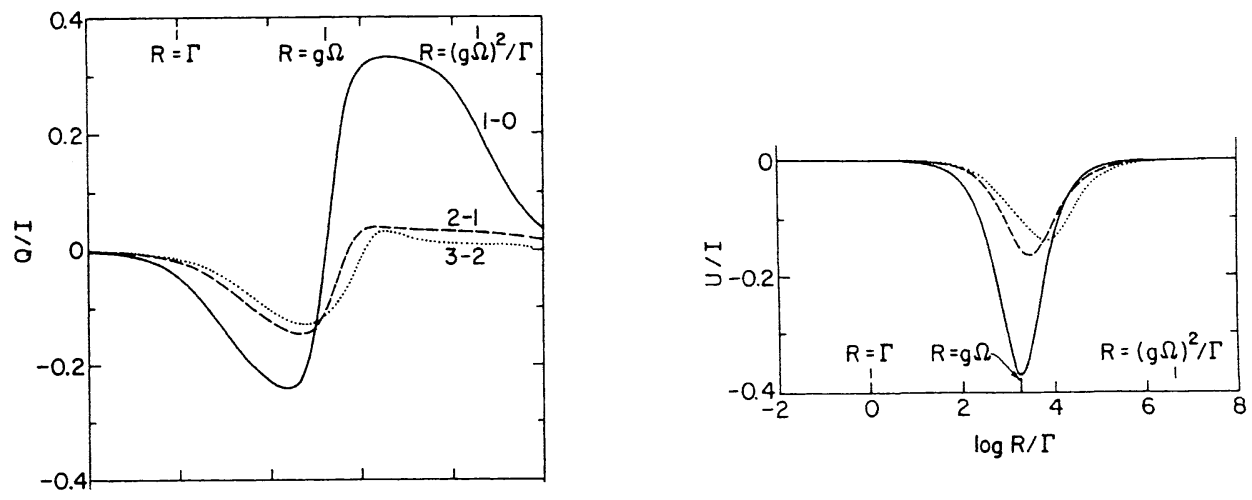

Figure 5. Fractional linear polarizations Stokes Q/I and U/I as a function of the degree of saturation for a linear maser in the regime in which the Zeeman splitting is much smaller than the spectral linebreadth. The results of calculations for angular momentum $J=1-0,2-1$, and 3-2 masing transitions are shown. The angle between the direction of the maser beam and the magnetic field is 45 degrees. As indicated in the figures, the ratio $g \Omega / \Gamma=2000$ is adopted in this example. From Nedoluha \& Watson (1990a).

anisotropic (or $m$-dependent) pumping of the magnetic substates is likely to be the most important. The absorption and/or the escape of infrared radiation by the masing molecules ordinarily is essential in the pumping of astrophysical masers. If the angular distribution of this radiation is anisotropic when it is incident upon the molecules, or if the angular distribution with which it escapes is anisotropic, then the magnetic substates of a molecular energy level will be pumped unequally. Explicit calculations for the radiative transfer of anisotropic, infrared radiation that is involved in the pumping were performed in the context of the circumstellar $\mathrm{SiO}$ masers to demonstrate that sufficient anisotropy can be present under conditions where the magnitude of the pumping is adequate to provide the maser power that is observed (Western \& Watson 1983). The circumstellar $\mathrm{SiO}$ masers are natural candidates for anisotropic pumping since they are located within a few stellar radii of the star. In addition, the medium in which they are located probably has strong velocity gradients which can create preferential directions for the escape of the infrared radiation. As a group, the circumstellar $\mathrm{SiO}$ masers do exhibit the highest fractional linear polarizations.

Even though anisotropic pumping associated with infrared radiation may be the dominant cause for the linear polarization, the direction of the polarization vector is still likely to be either parallel or perpendicular to the direction of the magnetic field as projected onto the surface of the sky (Nedoluha \& Watson 1990a). This will occur as long as $g \Omega$ is somewhat greater than $R$ and $\Gamma$ (except possibly when $R$ and $\Gamma$ are comparable) because the magnetic field is the dominant axis for symmetry. An analogous effect occurs in the alignment of 
interstellar dust grains. They are aligned either parallel or perpendicular to the magnetic field, regardless of the mechanism that causes the alignment, because the dust grains precess rapidly around the direction of the magnetic field. Faraday rotation within the maser can, however, reduce the magnitude and change the direction of the linear polarization just as it does for ordinary radiation (see Wallin \& Watson 1997 for an explicit calculation).

\section{Circular Polarization}

GKK considered only the polarization characteristics of the radiation at the center of the spectral line. In the weak splitting limit, no circular polarization occurs at line center.

The issues can be discussed in two ways. Firstly, to what degree is Stokes$\mathrm{V}$ due to what we consider to be the standard Zeeman effect altered by the saturation of a maser. Secondly, what are "non-Zeeman" effects that cause circular polarization. The distinction between these may not always be clear, in principle. In practice, it seems natural that "alterations to the standard Zeeman effect" should refer to changes that can be described in terms of the unequal populations of the magnetic substates that are created in saturated masers in the presence of a magnetic field when $\Gamma \ll R \ll g \Omega$. Non-Zeeman effects encompass everything else.

\section{C1. Alteration of Zeeman circular polarization}

Initial calculations of the influence of saturation on the standard Zeeman effect focussed on the $22 \mathrm{GHz}$ water transition (Nedoluha \& Watson 1992). The calculations were limited to saturations $R / \Gamma \leq 10$. They nevertheless demonstrated that the standard relationship $\mathrm{V} / \partial I / \partial \omega=$ constant $\times \mathrm{B} \cos \theta$ for twolevel transitions, which is applicable for thermal spectral lines and for masing lines that are unsaturated, is altered by the effects of saturation. At larger angles $\theta, \mathrm{V} /(\partial I / \partial \omega)$, was found be greater by a factor of 2 or so for the $\mathrm{F}=7$ 6 hyperfine transition than would be expected from the standard relationship. Since the $22 \mathrm{GHz}$ transition probably is a result of the merger of three hyperfine components, the standard relationship between $\mathrm{V}$ and $\partial I /(\partial \omega)$ most likely is meaningless Alternative relationships exist (e.g., Watson \& Wiebe 2001). More extensive calculations have now been performed (Watson \& Wyld, in preparation) for $J=1-0$ and $J=2-1$ masing transitions. The quantity $V / \partial I /(\partial \omega)$ is increased in these calculations beyond that given by the standard relationship by the effects of saturation. The magnitude of the increase is an increasing function of the angle $\theta$ until a maximum is reached at some large angle which depends somewhat on the degree of saturation. For $R / \Gamma \gtrsim 3, \mathrm{~V} /(\partial I / \partial \omega)$ increases, not decreases, with angle $\theta$ within this range of angles.

\section{C2. Non-Zeeman circular polarization}

Modifications to the standard Zeeman effect, which have been discussed above, are likely to cause an uncertainty of only a factor of a few in the inferred magnetic fields. In contrast, when they are effective, "non-Zeeman" mechanisms tend to create large circular polarization that is difficult to relate in a useful way to the strength of the magnetic field. Non-Zeeman circular polarization, as defined in the foregoing, occurs when the medium is anisotropic and the principal optical axes of the medium are not aligned with the direction of the linear polarization of radiation that is passing through the medium. The effect is 
analogous to the creation of circularly polarized radiation when ordinary, linearly polarized light passes through a sheet of polaroid material (or a "quarter-wave plate") with axes that are not aligned with the direction of the polarization of the light. In astronomy, this effect is observed to occur for optical light when the direction of the magnetic field, and hence the direction of alignment of the polarizing dust grains, changes along the line of sight. The two requirements are then-firstly, the masing medium must be optically anisotropic and secondly, its optical axes and the direction of the linear polarization of the radiation must be misaligned along the path of the radiation.

The masing medium will be anisotropic when the populations of the magnetic substates are unequal. They will be unequal when there is anisotropic pumping or when the linear polarization is created in the GKK mechanism. More generally, when linearly polarized radiation is created within a medium, it follows that the medium is anisotropic. The medium must be anisotropic to distinguish one direction from another-which is required to establish a direction for the linear polarization of the radiation. When linearly polarized radiation is observed, it then follows immediately that the first requirement for non-Zeeman circular polarization is satisfied unless the masing medium is simply amplifying background radiation that is already linearly polarized to the same degree that is observed. The masing medium is surely optically anisotropic in the spectral lines of the $\mathrm{SiO}$ masers which tend to be have high linear polarization.

Establishing when the second requirement for non-Zeeman circular polarization is satisfied is more difficult. When $g \Omega \gg R$ and $\Gamma$, the optical axes of the medium ordinarily will be aligned with the magnetic field. Thus, changes in the projected direction of the magnetic field on the plane of the sky within the masing gas will cause the optical axes and the direction of the linear polarization of the maser radiation to become misaligned. Using a statistical description of turbulent velocity and magnetic fields, we (Wiebe \& Watson 1998) have performed explicit computations to see what can be expected when the path lengths for masing and the changes in the direction of the magnetic field within these path lengths are determined by a plausible description of the medium. The characteristics of the linear and circular polarization that emerge in this calculation are similar to what is observed for the circumstellar $\mathrm{SiO}$ masers. Even in the absence of any change in the magnetic field, the optical axes and the direction of the linear polarization will become misaligned when $R \approx g \Omega$. This can easily be seen to occur, for example in Figure 5, where the direction of the linear polarization due to the GKK mechanism rotates by 90 degrees as $R$ approaches and significantly exceeds $g \Omega$. Creation of non-Zeeman circular polarization when $R \approx g \Omega$ has been investigated in considerable detail (Nedoluha \& Watson 1990b; 1994).

The conversion of linear to circular polarization in an anisotropic medium occurs because the phase velocity of a linearly polarized electromagnetic wave is different when its electric field is parallel to one, as opposed to the other, of the optical axes. Within a spectral line, however, which of the axes has the larger phase velocity is reversed at the center of the spectral line. Radiative transitions of molecules are analogous to classical resonances in making a contribution to both the real and imaginary parts of the dielectric constant of the medium (e.g., Jackson 1999). The imaginary part of the dielectric constant describes 
the absorption and emission due to the radiative transition. The contribution of the resonance to the real part of the dielectric constant determines the phase velocity of the medium at frequencies within the spectral line. This contribution is antisymmetric about line center, and hence a reversal occurs at line center as to which phase velocity is the larger. As a result, an antisymmetric Stokes-V is obtained when circularly polarized radiation is created by the non-Zeeman mechanisms being described here. The profile of this Stokes-V is quite similar to the profile that is expected when the Stokes- $\mathrm{V}$ is due to the standard Zeeman effect-at least for isolated, two-level transitions (e.g., Wiebe \& Watson 1998 for representative spectra).

Finally, assertions have been made that "fluctuations", "instabilities" and perhaps other ill defined, similar effects cause the polarization of masers to be quite different from the description in this Appendix. In all cases where such effects have been examined in a meaningful way for astrophysical masers, they are unimportant (GKK; Western 1983; Watson 1994; Wallin \& Watson 1995). Non-local instabilities do exist in the radiative transfer equations for astrophysical masers, but only in a quite limited region of parameter space (Scappaticci \& Watson 1992).

\section{References}

Anderson, N., \& Watson, W.D., 1993, ApJ, 407, 620

Baan, W.A., Haschick, A.D., \& Peng, E.W. 1993, BAAS, 25, 1325

Babkovskaya, N.S.,\& Varshalovich, D.A. 2000, Astronomy Letters, 26, 144

Blandford, R.D., \& Payne,D.G. 1982, MNRAS, 199, 883

Bragg, A.E., Greenhill, L.J., Moran, J.M., \& Henkel, C. 2000, ApJ, 535,73

Chary, R., Becklin, E.E., Evans, A.S., Neugebauer, G., Scoville, N.Z., Matthews, K., \& Ressler, M.E. 2000, ApJ, 531, 756

Collison, A.J., \& Watson, W.D. 1995, ApJ, 452, L103

Desch, S., Wallin, B.K., \& Watson, W.D. 1998, ApJ, 496,775

Deguchi, S. 1981, ApJ, 249,145

Deguchi, S. 1982, ApJ, 259, 634

Deguchi, S. 1994, ApJ, 420, 551

Deguchi, S., \& Watson, W.D. 1989, ApJ, 340, L17

Deguchi, S., \& Watson, W.D. 1990, ApJ, 354, 649

Emmering, R. T., \& Watson, W.D. 1994, ApJ, 495, 774

Gallimore, J.F., Henkel, C., Baum, S.E., Glass, I.S., Claussen, M.J., Prieto, M.A., \& van Kap-herr, A. 2001, ApJ, in press

Gangadhara,R.T., Deguchi, S., \& Lesch, H. 1999, Phys. Plasmas, 6, 4088

Gammie, C.F., Narayan, R., \& Blandford, R. 1999, ApJ, 516, 177

Goldreich, P., Keeley, D.A., \& Kwan, J.Y. 1973a, ApJ, 179,111 [GKK]

Goldreich, P., Keeley, D.A., \& Kwan, J.Y. 1973b, ApJ, 182, 55

Goldreich, P., \& Kwan, J.Y. 1974, ApJ, 190,27 
Herrnstein, J.R., Moran, J.M., Greenhill, L.J., Blackman, E.G.,\& Diamond, P.J. 1998, ApJ, 508,243

Jackson, J.D. 1999, Classical Electrodynamics (New York:Wiley),309

Kartje, J.F., Konigl, A., \& Elitzur, E. 1999, ApJ, 513, 180

Kumar, P. 1999, ApJ, 519, 599

Lasota, J.P. et al. 1996, ApJ.,462, 142

Maoz, E, \& McKee, C.F. 1998, ApJ, 494, 218

Miller, K.A., \& Stone, J.M. 2000, ApJ, 534, 398

Miyoshi, M., Moran, J., Herrnstein, J., Greenhill, L., Nakai, N., Diamond, P., \& Inoue, M. 1995, Nature, 373, 127

Moran, J.M., Greenhill, L.J., \& Herrnstein, J.R 1999, J. Ap. Astr., 20, 165

Moran, J.M.. 2000, Phil.Trans.R. Soc. Lond. A, 358,797

Nedoluha, G.E., \& Watson, W.D. 1990a, ApJ, 354,669

Nedoluha, G.E., \& Watson, W.D. 1990b, ApJ, 361,L53

Nedoluha, G.E., \& Watson, W.D. 1991, ApJ, 367, L63

Nedoluha, G.E., \& Watson, W.D. 1992, ApJ, 384,185

Nedoluha, G.E., \& Watson, W.D. 1994, ApJ, 423,394

Neufeld, D.N., Maloney, P.R., \& Conger, S. 1994, ApJ, 436, L127

Neufeld, D.N., \& Maloney, P.R. 1995, ApJ, 447, L17

Neufeld, D.N. 2000, ApJ, 542, L99

Scappaticci, G.A., \& Watson, W.D. 1992, ApJ, 400, 351

Schmeld, I.K., Strelnitskii, V.S., \& Muzylev, V.V. 1976, Astron. Zh., 53, 728

Sobolev, A.M., Wallin, B.K., \& Watson, W.D. 1998, ApJ, 498, 763

Stella, L., \& Rosner, R. 1984, ApJ, 277, 312

Wallin, B.K., \& Watson, W.D. 1995, ApJ, 445, 465

Wallin, B.K., \& Watson, W.D. 1997, ApJ, 481, 832

Wallin, B.K., Watson, W.D., \& Wyld, H.W. 1998, ApJ, 774,775

Wallin, B.K., Watson, W.D., \& Wyld, H.W. 1999, ApJ, 517,682

Watson, W.D. 1994, ApJ, 424, L37

Watson, W.D., \& Wallin, B. K. 1994, ApJ, 432, L35

Watson, W.D., \& Wallin, B. K. 1997, ApJ, 476, 685

Watson, W.D., \& Wiebe, D.S. 2001, ApJ, in press

Watson, W.D., \& Wyld, H.W. 2000, ApJ, 530, 207

Western, L.R. 1983, PhD dissertation, Univ. of Illinois

Western, L.R., \& Watson, W.D. 1983, ApJ, 275,195

Western, L.R., \& Watson, W.D. 1984, ApJ,285,158

Wiebe, D.S., \& Watson, W.D. 1998, ApJ, 503,L71 